\title{
Local attractivity for integro-differential equations with noncompact semigroups
}

https://doi.org/10.1515/msds-2020-0113

Received June 5, 2020; accepted September 23, 2020

Abstract: In this paper, we are devoted to study the existence and local attractivity of solutions for a class of integro-differential equations.Under the situation that the nonlinear term satisfy Carathéodory conditions and a noncompactness measure condition, we establish some existence and local attractivity of mild solutions by utilizing Mönch fixed point theorem, Kuratowski measure of noncompactness and resolvent operator theory in the sense of Grimmer.Our investigations will be situated in the Banach space of real functions which are defined, continuous, and bounded on the right-hand real half axis $\mathbb{R}^{+}$. Moreover an example is given to illustrate our outcomes.

Keywords: Integro-differential equations, Mild solution, Measure of noncompactness, Resolvent operator, Attractivity

MSC: 45D05, 34G20, 47J35

\section{Introduction}

Evolution equations are used in many fields of applied mathematics[9, 33].In recent years, this type of equation has gained a lot of attention[39].The theory of integro-differential equations on infinite intervals plays a key role as it addresses certain real world problems; it has gained considerable attention recently and now represents an important nonlinear analysis branch. Broad applications for physics, mechanics, engineering, electric, chemistry, economy and other areas $[2,6,35,36]$ have, in recent years, been found in integrodifferential equations; while numerous research papers and monographs have been written, devoting themselves to functional and integro-differential equations[3, 4, 7, 21, 31, 34, 37]. Such documents contain specific qualitative properties like existence, uniqueness, stability and asymptotic behavior of first-order equations. On the other hand measure of noncompactness is one of the most useful tools in nonlinear and functional analysis, metric fixed point theory and the theory of operator equations in Banach spaces which was first introduced by Kuratowski in[28]. This concept also used to investigate functional equation, ordinary and partial differential equations, integral and integro-differential equations. In this context several authors have pre-

Amadou Diop: Université Gaston Berger de Saint-Louis, UFR SAT, Département de Mathématiques, B.P. 234, Saint-Louis, Sénégal, E-mail: diop.amadou@ugb.edu.sn

*Corresponding Author: Mamadou Abdoul Diop: Université Gaston Berger de Saint-Louis, UFR SAT, Département de Mathématiques, B.P. 234, Saint-Louis, Sénégal,

UMMISCO UMI 209 IRD/UPMC, Bondy, France, E-mail: mamadou-abdoul.diop@ugb.edu.sn

Ouaténi Diallo: Université des Sciences, des Techniques et des Technologies de Bamako, Ecole Doctorale des Sciences et Technologies du Mali, B.P. E2528, Bamako, Mali, E-mail: ouateni@yahoo.fr

Mariam B Traoré: Université des Sciences, des Techniques et des Technologies de Bamako, Ecole Doctorale des Sciences et Technologies du Mali, B.P. E2528, Bamako, Mali, E-mail: traoremariam34@yahoo.fr 
sented some papers on the existence of solution for nonlinear integral equations which involves the use of measure of noncompactness and many other techniques, for instance see[15, 16] and [10,11].

Motivated by the above discussion, in this paper we consider the following integro-differential equation of the form

$$
\left\{\begin{array}{l}
\vartheta^{\prime}(t)=\mathrm{A} \vartheta(t)+\int_{0}^{t} Y(t-r) \vartheta(r) d r+\mathrm{F}\left(t, \vartheta(t), \int_{0}^{t} \rho(t, r, \vartheta(r)) d r\right), \quad t \in I=[0,+\infty), \\
\vartheta(0)=\vartheta_{0},
\end{array}\right.
$$

where $A$ is the infinitesimal generator of a strongly semigroup $(\mathrm{T}(\mathrm{t}))_{t \geq 0}$ on a Banach space $\mathbf{W}$ with domain $\mathscr{D}(\mathrm{A})$. Here $Y(t)$ is a closed linear operator on $\mathbf{W}$, with domain $\mathscr{D}(\mathrm{A}) \subset \mathscr{D}(Y(t))$, which is independent of $t$. $\mathrm{F}$ : $I \times \mathbf{W} \times \mathbf{W} \rightarrow \mathbf{W}$ is a Carathéodory function, $\rho: \Delta \times \mathbf{W} \rightarrow \mathbf{W}$ is a continuous function, $\Delta:=\{(t, s) \in I \times I$ : $s \geq t\}, \vartheta_{0} \in \mathbf{W}$, and $(\mathbf{W},\|\cdot\|)$ is a real Banach space.

Moreover, up to now no work has been reported yet regarding the local attractivity for a class of integro-differential equations, which motivates this present study. The main contributions of this paper are summarized as follows:

- In this work, a general class of integro-differential equation is considered.

- Using methods of functional analysis, a set of sufficient conditions are proposed for ensuring the existence and local attractivity of mild solutions.

- The results are established with the use of the theory of resolvent operator in the sense of Grimmer and Kuratowski measure of noncompactness.We use the fact that the operator-norm continuity of the resolvent operator is equivalent to that of the semigroup generated by the coefficient operator. This property allows us to drop the supposition that the operator semigroup is compact and to show that the operator solution meets the requirements of the Mönch conditions.

- The result in this paper generalizes and improves some of previous ones in this field.Our paper expands the usefulness of integro-differential equations, since the literature shows results for existence and attractivity for such equations in the case of semigroup only.

The rest of our paper is as follows.In the next section, we recall some preliminaries about the resolvent operator theory, measure of noncompactness and the fixed point theory for condensing maps. Section 3 is devoted to the existence result under a general setting via measure of noncompactness. Section 4 shows the attractivity of the solution to the problem (1.1). The last section gives an example, which illustrates the feasibility of the abstracts results obtained in the paper.

\section{Preliminaries}

In this section, we collect some notations, definitions and supplementary informations that are included in the further considerations of this article.Throughout the paper, $\mathbf{W}$ is a Banach space, $\mathbf{A}$ and $Y(t)$ are closed linear operators on $\mathbf{W} . \mathscr{B}$ represents the Banach space $D(A)$ equipped with the graph norm

$$
\|\vartheta\|_{\mathscr{B}}:=\|\mathrm{A} \vartheta\|+\|\vartheta\| \text { for } \vartheta \in \mathscr{B} .
$$

The notations $\mathrm{BC}(\mathrm{I}, \mathbf{W}), \mathscr{C}\left(\mathbb{R}^{+}, \mathscr{B}\right)$ stand for the Banach space of all bounded and continuous functions $\vartheta$ mapping $I$ into $\mathbf{W}$, the space of all functions from $\mathbb{R}^{+}$into $\mathscr{B}$ which are continuous. 
For any constant $q>0$, let

$$
B_{q}=\{\vartheta \in \mathrm{BC}(\mathrm{I}, \mathbf{W}):\|\vartheta\|<q\}, \bar{B}_{q}=\{\vartheta \in \mathrm{BC}(\mathrm{I}, \mathbf{W}):\|\vartheta\| \leq q\} .
$$

We recall some knowledge on partial integro-differential equations and the related resolvent operators. Let us consider the following system for further purposes :

$$
\left\{\begin{array}{l}
\vartheta^{\prime}(t)=\mathrm{A} \vartheta(t)+\int_{0}^{t} Y(t-s) \vartheta(s) d s \text { for } t \geq 0, \\
\vartheta(0)=\vartheta_{0} \in \mathscr{B} .
\end{array}\right.
$$

Definition 2.1. ([38]) A resolvent operator for Eq. (2.1) is a bounded linear operator valued function $\mathrm{R}(t) \in$ $\mathscr{L}(\mathbf{W})$ for $t \geq 0$, having the following properties :

(i) $\mathrm{R}(0)=I$ (the identity map of $\mathbf{W}$ ) and $\|\mathrm{R}(t)\| \leq N e^{\beta t}$ for some constants $N>0$ and $\beta \in \mathbb{R}$

(ii) For each $\vartheta \in \mathbf{W}, \mathrm{R}(t) \vartheta$ is strongly continuous for $t \geq 0$.

(iii) For $\vartheta \in \mathbf{W}, \mathrm{R}(.) \vartheta \in \mathscr{C}^{1}\left(\mathbb{R}^{+} ; \mathbf{W}\right) \cap \mathscr{C}\left(\mathbb{R}^{+} ; \mathscr{B}\right)$ and

$$
\begin{aligned}
\mathrm{R}^{\prime}(t) \vartheta & =\operatorname{AR}(t) \vartheta+\int_{0}^{t} Y(t-s) \mathrm{R}(s) \vartheta d s \\
& =\mathrm{R}(t) \mathrm{A} \vartheta+\int_{0}^{t} \mathrm{R}(t-s) Y(s) \vartheta d s, \text { for } t \geq 0 .
\end{aligned}
$$

In what follows, we make the following assumptions.

$\left(\mathbf{H}_{1}\right)$ The operator $A$ is the infinitesimal generator of a strongly continuous semigroup $(\mathrm{T}(t))_{t \geq 0}$ on $\mathbf{W}$.

$\left(\mathbf{H}_{2}\right)$ For all $t \geq 0, Y(t)$ is closed linear operator from $D(\mathrm{~A})$ to $\mathbf{W}$ and $Y(t) \in \mathscr{L}(\mathscr{B}$, W). For any $\vartheta \in \mathbf{W}$, the map $t \mapsto Y(t) \vartheta$ is bounded, differentiable and the derivative $t \mapsto Y^{\prime}(t) \vartheta$ is bounded and uniformly continuous on $\mathbb{R}^{+}$.

The following theorem provides adequate conditions to ensure that the resolvent operator for equation (2.1) exists.

Theorem 2.1 ([38]). Assume that $\left(\mathbf{H}_{1}\right)-\left(\mathbf{H}_{2}\right)$ hold. Then there exists a unique resolvent operator to the Cauchy problem (2.1).

In the following, we give some results for the existence of solutions for the following integro-differential equation.

$$
\left\{\begin{array}{l}
\vartheta^{\prime}(t)=\mathrm{A} \vartheta(t)+\int_{0}^{t} Y(t-s) \vartheta(s) d s+l(t) \text { for } t \geq 0, \\
\vartheta(0)=\vartheta_{0} \in \mathscr{B},
\end{array}\right.
$$

where $l:[0,+\infty[\rightarrow \mathscr{B}$ is a continuous function.

Definition $2.2([38])$. A continuous function $\vartheta:[0,+\infty[\rightarrow \mathbf{W}$ is said to be a strict solution of the Eq. (2.2) if

1. $\vartheta \in \mathscr{C}^{1}([0,+\infty[, \mathbf{W}) \cap \mathscr{C}([0,+\infty[, \mathscr{B})$,

2. $\vartheta$ satisfies Eq. (2.2) for $t \geq 0$. 
Theorem 2.2 ([38]). Assume that hypotheses $\left(\mathbf{H}_{\mathbf{1}}\right)$ and $\left(\mathbf{H}_{2}\right)$ hold. If $\vartheta$ is a strict solution of the Eq.(2.2), then the variation of constant formula holds

$$
\vartheta(t)=\mathrm{R}(t) \vartheta_{0}+\int_{0}^{t} \mathrm{R}(t-s) l(s) d s \quad \text { for } t \geq 0 .
$$

We recall the following theorem which sets the equivalence between the $C_{0}$-semigroup operator-norm continuity and the integral equations resolvent operator.

Theorem 2.3 ([25]). Let A be the infinitesimal generator of a $C_{0}$-semigroup $(\mathrm{S}(t))_{t \geq 0}$ and let $(Y(t))_{t \geq 0}$ satisfy $\left(\mathbf{H}_{\mathbf{2}}\right)$. Then the resolvent operator $(\mathrm{R}(t))_{t \geq 0}$ for equation (2.2) is operator-norm continuous (or continuous in the uniform operator topology) for $t>0$ if and only if $(\mathrm{T}(t))_{t \geq 0}$ is operator-norm continuous for $t>0$.

We remember some characteristics of the measure of noncompactness and the Mönch fixed-point theorem to prove the paper's primary outcome.

Definition 2.3 ([12]). Let D be a bounded subset in any Banach space Y. The Kuratowski measure of noncompactness (shortly $M N C$ ) is defined by

$$
\alpha(D)=\inf \{\epsilon>0: \text { D has a finite cover by sets of diameter } \leq \epsilon\} .
$$

Let us recall the basic properties of Kuratowski measure of noncompactness.

Lemma 2.4. [12] Let $\mathrm{Y}$ be a Banach space and $\mathrm{D}_{1}, \mathrm{D}_{2}$ be bounded subset of $\mathrm{Y}$, then the following properties hold:

1. $\alpha\left(\mathrm{D}_{1}\right) \leq \alpha\left(\mathrm{D}_{2}\right)$ when $\mathrm{D}_{1} \subset \mathrm{D}_{2}$,

2. $\alpha\left(\mathrm{D}_{2}\right)=\alpha\left(\overline{\mathrm{D}_{2}}\right), \overline{\mathrm{D}_{2}}$ denotes the closure of $\mathrm{D}_{2}$,

3. $\alpha\left(\mathrm{D}_{1}+\mathrm{D}_{2}\right) \leq \beta\left(\mathrm{D}_{1}\right)+\beta\left(\mathrm{D}_{2}\right)$ where $\mathrm{D}_{1}+\mathrm{D}_{2}=\left\{x+y: x \in \mathrm{D}_{1}, y \in \mathrm{D}_{2}\right\}$,

4. $\alpha\left(\lambda \mathrm{D}_{2}\right) \leq|\lambda| \beta\left(\mathrm{D}_{2}\right)$ for any $\lambda \in \mathbb{R}$,

5. $\alpha\left(\{a\} \cup \mathrm{D}_{1}\right)=\alpha\left(\mathrm{D}_{1}\right)$ for any $a \in \mathrm{Y}$,

6. $\alpha\left(\mathrm{D}_{2}\right)=0$ if and only if $\mathrm{D}_{2}$ is relatively compact,

7. $\alpha\left(\mathrm{D}_{2}\right)=\alpha\left(\overline{\operatorname{Conv}}\left(\mathrm{D}_{2}\right)\right)$, where $\overline{\operatorname{Conv}}\left(\mathrm{D}_{2}\right)$ is closed convex hull of $\mathrm{D}_{2}$,

8. $\alpha\left(\mathrm{D}_{1} \cup \mathrm{D}_{2}\right)=\max \left(\alpha\left(\mathrm{D}_{1}\right), \alpha\left(\mathrm{D}_{2}\right)\right)$.

Denote by $\omega^{T}(\vartheta, \epsilon)$ the modulus of continuity of $\vartheta$ on the interval $[0, T]$ i.e

$$
\omega^{T}(\vartheta, \epsilon)=\sup \{\|\vartheta(t)-\vartheta(s)\| ; t, s \in[0 ; T],|t-s| \leq \epsilon\} .
$$

Moreover, let us put

$$
\begin{aligned}
\omega^{T}(B, \epsilon) & =\sup \left\{\omega^{T}(\vartheta, \epsilon) ; \vartheta \in B\right\}, \\
\omega_{0}^{T}(B) & =\lim _{\epsilon \rightarrow 0} \omega^{T}(B, \epsilon) .
\end{aligned}
$$

For more details about properties of $\omega_{0}^{T}$ (.), one can see [29]. The next results play an important role in demonstrating our key findings.

Lemma 2.5 ([22]). If $\mathbb{U}=\left\{\mathrm{u}_{n}\right\}_{n=0}^{\infty} \subset L^{1}([0 ; b], W)$ is uniformly integrable, then the function $s \rightarrow \alpha(\mathbb{U}(s))$ is measurable and

$$
\alpha\left\{\int_{0}^{t} \mathrm{u}_{n}(s) d s\right\}_{n=0}^{\infty} \leq 2 \int_{0}^{t} \alpha(\mathbb{U}(s)) d s, \quad t \in[0, T] .
$$


Remark 2.1. A subset $\mathbb{X} \subset L^{1}([0 ; T], W)$ is uniformly integrable if there exists $\mu \in L^{1}\left([0 ; T] ; \mathbb{R}^{+}\right)$such that

$$
\|x(t)\| \leq \mu(t) \text { for } x \in \mathbb{X} \text { and a.e. } s \in[0 ; T] .
$$

Lemma 2.6 ([5]. p. 35). Let $A(t), B(t), C(t)$ and $D(t)$ be real valued nonnegative integrable functions on $[0,+\infty)$, for which the inequality

$$
A(t) \leq B(t)+\int_{0}^{t} C(s)\left[A(s)+\int_{0}^{s} D(\tau) A(\tau) d \tau\right] d s
$$

holds for all $t \in[0 ;+\infty)$, then

$$
A(t) \leq B(t)+\int_{0}^{t} C(s)\left[B(s)+\int_{0}^{t} B(\tau)(C(\tau)+D(\tau)) \exp \left(\int_{\tau}^{s}(C(\delta)+D(\delta)) d \delta\right) d \tau\right] d s
$$

for all $t \in[0 ;+\infty)$.

We introduce now the concept of attractivity (stability) of solutions of operator equations in the space $\mathrm{BC}(\mathrm{I}, \mathbf{W})$. To this end, assume that $\mathscr{F}$ is a nonempty subset of the space $\mathrm{BC}(\mathrm{I}, \mathbf{W})$. Moreover, let $\Pi$ be an operator defined on $\mathscr{F}$ with values in $\mathrm{BC}(\mathrm{I}, \mathbf{W})$. Let us consider the operator equation of the form

$$
\vartheta(t)=(\Pi \vartheta)(t) .
$$

Definition 2.4. We say that solutions of (1.1) are locally attractive if there exists a ball $B\left(\vartheta^{\star}, \delta\right)$ in the space $\mathrm{BC}(\mathrm{I}, \mathrm{W})$ such that $\bar{B}\left(\vartheta^{\star}, \delta\right) \cap \mathscr{F} \neq 0$, and for arbitrary solutions $\vartheta^{(1)}$ and $\vartheta^{(2)}$ of $(2.4)$ belonging to $\bar{B}\left(\vartheta^{\star}, \delta\right) \cap \mathscr{F}$ we have

$$
\lim _{t \rightarrow+\infty}\left(\vartheta^{(1)}(t)-\vartheta^{(2)}(t)\right)=0 .
$$

We will say that solutions of (2.4) are uniformly locally attractive when for each $\epsilon>0$ there exists $a T>0$ such that

$$
\left\|\vartheta^{(1)}(t)-\vartheta^{(2)}(t)\right\| \leq \epsilon
$$

for all $\vartheta^{(1)}, \vartheta^{(2)} \in \bar{B}\left(\vartheta^{\star}, \delta\right) \cap \mathscr{F}$ being solutions of (2.4) and for $t \geq T$.

The following nonlinear fixed point Theorem of Mönch plays an significant role in proving our primary outcome.

Lemma 2.7. [8] Let $X$ be a Banach space, $\mathscr{O}$ is bounded open subset of $X$ with $0 \in \mathscr{O}$. Let $\mathrm{F}: \overline{\mathscr{O}} \rightarrow X$ be a continuous operator satisfying

(i) If $K \subset \overline{\mathscr{O}}$ is countable and $K \subset \overline{\operatorname{Conv}}(\{0\} \cup \mathrm{F}(K))$; then $K$ is relatively compact.

(ii) $\vartheta \neq \kappa \mathrm{F} \vartheta ; \quad \forall \kappa \in[0 ; 1]: \quad \vartheta \in \partial \mathscr{O}$,

Then $\mathrm{F}$ has a fixed point in $\overline{\mathscr{O}}$.

\section{Existence of mild solutions}

Definition 3.1. A function $\vartheta(\cdot) \in \mathrm{BC}(\mathrm{I}, \mathbf{W})$ is said to be a mild solution of the system (1.1) if

$$
\vartheta(t)=\mathrm{R}(t) \vartheta_{0}+\int_{0}^{t} \mathrm{R}(t-s) \mathrm{F}\left(s, \vartheta(s), \int_{0}^{t} \varrho(s, \tau, \vartheta(\tau)) d \tau\right) d s, \text { for } t \geq 0 .
$$


The following hypotheses will be further considered in order to present and show the existence outcomes for the issue (1.1).

$\left(\mathbf{C}_{1}\right)$ The semigroup $(\mathrm{T}(t))_{t \geq 0}$ is norm continuous for $t>0$.

$\left(\mathbf{C}_{2}\right)$ There exist constants $\mathrm{m}_{0} \geq 1$ and $\gamma>0$ satisfying

$$
\|\mathrm{R}(t)\|_{\mathscr{L}(\mathbf{W})} \leq \mathrm{m}_{0} e^{-\gamma t} \text { for } t \geq 0 .
$$

$\left(\mathbf{C}_{3}\right)$ The function $\mathrm{F}: I \times \mathbf{W} \times \mathbf{W} \rightarrow \mathbf{W}$ is Carathéodory and satisfies the following conditions :

(i)

$$
\lim _{t \rightarrow+\infty} \int_{0}^{t} e^{-\gamma(t-r)}\|\mathrm{F}(r, 0,0)\| d r=0
$$

(ii) There exists an integrable function $f: I \rightarrow \mathbb{R}^{+}$, such that :

$$
\left\|\mathrm{F}\left(t, \Phi_{2}, \Psi_{2}\right)-\mathrm{F}\left(t, \Phi_{1}, \Psi_{1}\right)\right\| \leq f(t)\left(\left\|\Phi_{2}-\Phi_{1}\right\|+\left\|\Psi_{2}-\Psi_{1}\right\|+1\right)
$$

for a.e $t \in I$ and each $\Phi_{i}, \Psi_{i} \in W,(i=1,2)$ and

$$
\lim _{t \rightarrow+\infty} \int_{0}^{t} e^{-\gamma(t-r)} \mathrm{f}(r) d r=0
$$

(iii) There exist locally integrable functions $\omega_{i}: I \rightarrow \mathbb{R}^{+},(i=1,2)$ such that :

$$
\alpha\left(\mathrm{F}\left(t, E_{1}, E_{2}\right)\right) \leq \omega_{1}(t) \alpha\left(E_{1}\right)+\omega_{2}(t) \alpha\left(E_{2}\right)
$$

for a.e $(t, s) \in \Delta$ and $E_{1}, E_{2} \subset \mathbf{W}$.

$\left(\mathbf{C}_{4}\right)$ The function $\rho: \Delta \times \mathbf{W} \rightarrow \mathbf{W}$ satisfies the following conditions:

(i) There exists an integrable function $g: I \rightarrow \mathbb{R}^{+}$, such that:

$$
\left\|\rho\left(t, s, \vartheta_{1}\right)-\rho\left(t, s, \vartheta_{2}\right)\right\| \leq g(s)\left\|\vartheta_{1}-\vartheta_{2}\right\| \text {, for a.e }(t, s) \in \Delta \text { and each } \vartheta_{1}, \vartheta_{2} \in \mathbf{W}
$$

(ii) There exist constants $M \geq 0$ and $\omega>0$, such that

$$
\|\rho(t, s, 0)\|<M e^{-\omega(t-s)} \text { for any }(t, s) \in \Delta .
$$

(iii) There exists constants $M^{*}>0$, such that

$$
\alpha(\rho(t, s, B)) \leq M^{\star} \alpha(B) \text { for a.e }(t, s) \in \Delta \text { and } B \subset \mathbf{W} .
$$

Remark 3.1. Notice that if the hypothesis $\left(\mathbf{C}_{3}\right)$ holds, then there exist constants $\bar{F}, \bar{f}>0$ such that :

$$
\overline{\mathrm{F}}=\sup _{t \in I} \int_{0}^{t} e^{-\gamma(t-r)} \mathrm{F}(r, 0,0) d r \quad \text { and } \quad \overline{\mathrm{f}}=\sup _{t \in I} \int_{0}^{t} e^{-\gamma(t-r)} \mathrm{f}(r) d r .
$$

Theorem 3.1. Assume that conditions $\left(\mathbf{H}_{1}\right)-\left(\mathbf{H}_{2}\right),\left(\mathbf{C}_{2}\right)-\left(\mathbf{C}_{4}\right)$ hold and equation (1.1) has a resolvent operator $(\mathrm{R}(t))_{t \geq 0}$ that is continuous in the operator norm topology for $t>0$. Then equation (1.1) has at least one solution.

Proof. Consider the operator $\Gamma: \mathrm{BC}(\mathrm{I}, \mathbf{W}) \rightarrow \mathrm{BC}(\mathrm{I}, \mathbf{W})$ defined by

$$
(\Gamma \vartheta)(t)=\mathrm{R}(t) \vartheta_{0}+\int_{0}^{t} \mathrm{R}(t-s) \mathrm{F}\left(s, \vartheta(s), \int_{0}^{t} \varrho(s, \tau, \vartheta(\tau)) d \tau\right) d s, \quad t \geq 0 .
$$

Observe that the fixed points of the operator $\Gamma$ are mild solutions of the problem (1.1). 
Step 1. $\Gamma(\vartheta) \in \mathrm{BC}(\mathrm{I}, \mathbf{W})$ for any $\vartheta \in \mathrm{BC}(\mathrm{I}, \mathbf{W})$.

Let $\vartheta \in \mathbf{W}$, then for $t \in I$, we get

$$
\begin{aligned}
& \|(\Gamma \vartheta)(t)\| \\
& =\left\|\mathrm{R}(t) \vartheta_{0}+\int_{0}^{t} \mathrm{R}(t-s) \mathrm{F}\left(s, \vartheta(s), \int_{0}^{s} \varrho(s, \tau, \vartheta(\tau)) d \tau\right) d s\right\| \\
& \leq \mathrm{m}_{0}\left\|\vartheta_{0}\right\|+\int_{0}^{t}\|\mathrm{R}(t-s)\|_{\mathscr{L}(\mathbf{W})}\left\|\mathrm{F}\left(s, \vartheta(s), \int_{0}^{s} \varrho(s, \tau, \vartheta(\tau)) d \tau\right)\right\| d s \\
& \leq \mathrm{m}_{0}\left\|\vartheta_{0}\right\|+\mathrm{m}_{0} \int_{0}^{t} e^{-\gamma(t-s)}\left\|\mathrm{F}\left(s, \vartheta(s), \int_{0}^{s} \varrho(s, \tau, \vartheta(\tau)) d \tau\right)-\mathrm{F}(s, 0,0)+\mathrm{F}(s, 0,0)\right\| d s \\
& \leq \mathrm{m}_{0}\left\|\vartheta_{0}\right\|+\mathrm{m}_{0} \int_{0}^{t} e^{-\gamma(t-s)} \mathrm{f}(s)\left(\|\vartheta(s)\|+\int_{0}^{s}\|\varrho(s, \tau, \vartheta(\tau))-\varrho(s, \tau, 0)+\varrho(s, \tau, 0)\| d \tau+1\right) d s \\
& +\mathrm{m}_{0} \int_{0}^{t} e^{-\gamma(t-s)}\|\mathrm{F}(s, 0,0)\| d s \\
& \leq \mathrm{m}_{0}\left\|\vartheta_{0}\right\|+\mathrm{m}_{0} \int_{0}^{t} e^{-\gamma(t-s)} \mathrm{f}(s)\left(\|\vartheta(s)\|+\int_{0}^{s}\|\varrho(s, \tau, \vartheta(\tau))-\varrho(s, \tau, 0)\| d \tau+1\right) d s \\
& \mathrm{~m}_{0} \int_{0}^{t} e^{-\gamma(t-s)} \mathrm{f}(s)\left(\int_{0}^{s}\|\varrho(s, \tau, 0)\| d \tau\right) d s+\mathrm{m}_{0} \int_{0}^{t} e^{-\gamma(t-s)}\|\mathrm{F}(s, 0,0)\| d s \\
& \leq \mathrm{m}_{0}\left\|\vartheta_{0}\right\|+\mathrm{m}_{0} \int_{0}^{t} e^{-\gamma(t-s)} \mathrm{f}(s)\left(\|\vartheta(s)\|+\int_{0}^{s} g(\tau)\|\vartheta(\tau)\| d \tau+1\right) d s \\
& +\mathrm{m}_{0} \int_{0}^{t} \int_{0}^{s} e^{-\gamma(t-s)} \mathrm{f}(s)\|\varrho(s, \tau, 0)\| d \tau d s+\mathrm{m}_{0} \int_{0}^{t} e^{-\gamma(t-s)}\|\mathrm{F}(s, 0,0)\| d s \\
& \leq \mathrm{m}_{0}\left\|\vartheta_{0}\right\|+\mathrm{m}_{0} \overline{\mathrm{f}}\left(\sup _{t \in I}\|\vartheta(t)\|+\|g\|_{L^{1}} \sup _{t \in I}\|\vartheta(t)\|+1\right)+\mathrm{m}_{0} \overline{\mathrm{f}} \int_{0}^{s}\|\varrho(s, \tau, 0)\| d \tau+\mathrm{m}_{0} \overline{\mathrm{F}} \\
& \leq \mathrm{m}_{0}\left\|\vartheta_{0}\right\|+\mathrm{m}_{0} \overline{\mathrm{f}}\left(1+\|g\|_{L^{1}}\right) \sup _{t \in I}\|\vartheta(t)\|+\mathrm{m}_{0} \overline{\mathrm{f}}\left(1+\frac{M}{\omega}\right)+\mathrm{m}_{0} \overline{\mathrm{F}} \\
& <+\infty \text {. }
\end{aligned}
$$

We deduce that, $\Gamma(\vartheta) \in \mathrm{BC}(\mathrm{I}, \mathbf{W})$.

Step 2. $\Gamma$ is continuous.

Let $\left(\vartheta^{(n)}\right)_{n \in \mathbb{N}}$ be a sequence in BC(I, W) such that $\vartheta_{n} \rightarrow \vartheta$ in BC $(\mathrm{I}, \mathbf{W})$. We consider two cases.

Case1. If $t \in[0, T], T>0$, then, we have

$$
\begin{aligned}
&\left\|\left(\Gamma \vartheta^{(n)}\right)(t)-(\Gamma \vartheta)(t)\right\|= \| \int_{0}^{t} \mathrm{R}(t-s) \mathrm{F}\left(s, \vartheta^{(n)}(s), \int_{0}^{s} \varrho\left(s, \tau, \vartheta^{(n)}(\tau) d \tau\right) d s\right. \\
&-\int_{0}^{t} \mathrm{R}(t-s) \mathrm{F}\left(s, \vartheta(s), \int_{0}^{s} \varrho(s, \tau, \vartheta(\tau)) d \tau\right) d s \| \\
& \leq \mathrm{m}_{0} \int_{0}^{t} \| \mathrm{F}\left(s, \vartheta^{(n)}(s), \int_{0}^{s} \varrho\left(s, \tau, \vartheta^{(n)}(\tau)\right) d \tau\right) \\
&-\mathrm{F}\left(s, \vartheta(s), \int_{0}^{s} \varrho(s, \tau, \vartheta(\tau)) d \tau\right) \| d s .
\end{aligned}
$$


Hence, since the function $F$ is Caratheodory and $\varrho$ is continuous function, the Lebesgue dominated convergence Theorem implies that $\left\|\Gamma\left(\vartheta^{(n)}\right)-\Gamma(\vartheta)\right\| d s \rightarrow 0$ as $n \rightarrow+\infty$.

Case 2. If $t \in(T,+\infty), T>0$.

Since $\vartheta^{(n)} \rightarrow \vartheta$ as $n \rightarrow \infty$, we conclude that for $\epsilon>0$, there is a real number $\bar{T} \geq 0$ such that

$$
\left\|\vartheta^{(n)}(t)-\vartheta(t)\right\| \leq \epsilon, \text { for any } t \geq \bar{T} .
$$

We choose $T \geq \bar{T}$, then

$$
\begin{aligned}
& \left\|\left(\Gamma \vartheta^{(n)}\right)(t)-(\Gamma \vartheta)(t)\right\| \\
= & \| \int_{0}^{t} \mathrm{R}(t-s) \mathrm{F}\left(s, \vartheta_{s}^{(n)}+\tilde{\kappa}_{s}, \int_{0}^{s} \varrho\left(s, \tau, \vartheta^{(n)}(\tau)\right) d \tau\right) d s \\
& \quad-\int_{0}^{t} \mathrm{R}(t-s) \mathrm{F}\left(s, \vartheta(s), \int_{0}^{s} \varrho(s, \tau, \vartheta(\tau)) d \tau\right) d s \| \\
\leq & \mathrm{m}_{0} \int_{0}^{t} e^{-\gamma(t-s)} \| \mathrm{F}\left(s, \vartheta_{s}^{(n)}+\tilde{\kappa}_{s}, \int_{0}^{s} \varrho\left(s, \tau, \vartheta^{(n)}(\tau)\right) d \tau\right) \\
\leq & \mathrm{m}_{0} \int_{0}^{t} e^{-\gamma(t-s)} f(s)\left[\left\|\vartheta^{(n)}(s)-\vartheta(s)\right\|+\int_{0}^{s} g(\tau)\left\|\vartheta^{(n)}(\tau)-\vartheta(\tau)\right\| d \tau+1\right] d s \\
\leq & \mathrm{m}_{0} \int_{0}^{t} e^{-\gamma(t-s)} f(s)\left[\epsilon\left(1+\|g\|_{L^{1}}\right)+1\right] d s .
\end{aligned}
$$

By hypothesis $\left(\mathbf{C}_{3}\right)$ - (ii) the above inequality, reduces to

$$
\left\|\Gamma \vartheta^{(n)}-\Gamma \vartheta\right\| \rightarrow 0 \text { as } n \rightarrow \infty .
$$

This implies that $\Gamma$ is continuous.

Step $3 \Gamma\left(B_{q}\right)$ is equicontinuous.

Let $t_{1}, t_{2} \in[0, T]$ with $t_{1}<t_{2}$ and $\theta \in B_{q}$. Then, we have

$$
\begin{aligned}
& \left\|(\Gamma \vartheta)\left(t_{2}\right)-(\Gamma \vartheta)\left(t_{1}\right)\right\| \\
\leq & \left\|\mathrm{R}\left(t_{2}\right) \vartheta_{0}-\mathrm{R}\left(t_{1}\right) \vartheta_{0}\right\|+\int_{0}^{t_{1}}\left\|\mathrm{R}\left(t_{2}-s\right)-\mathrm{R}\left(t_{1}-s\right)\right\| \mathscr{L}(\mathbf{W})\left\|\mathrm{F}\left(s, \vartheta(s), \int_{0}^{s} \varrho(s, \tau, \vartheta(\tau)) d \tau\right)\right\| d s \\
& +\int_{t_{1}}^{t_{2}}\left\|\mathrm{R}\left(t_{2}-s\right)\right\| \mathscr{L}(\mathbf{W})\left\|\mathrm{F}\left(s, \vartheta(s), \int_{0}^{s} \varrho(s, \tau, \vartheta(\tau)) d \tau\right)\right\| d s \\
\leq \quad & \left\|\mathrm{R}\left(t_{2}\right) \vartheta_{0}-\mathrm{R}\left(t_{1}\right) \vartheta_{0}\right\| \\
& +\int_{0}^{t_{1}}\left\|\mathrm{R}\left(t_{2}-s\right)-\mathrm{R}\left(t_{1}-s\right)\right\| \mathscr{L}(\mathbf{W}) \\
& \times\left(\left\|\mathrm{F}\left(s, \vartheta(s), \int_{0}^{s} \varrho(s, \tau, \vartheta(\tau)) d \tau\right)+\mathrm{F}(s, 0,0)-\mathrm{F}(s, 0,0)\right\|\right) d s \\
& +\int_{t_{1}}^{t_{2}}\left\|\mathrm{R}\left(t_{2}-s\right)\right\|\left\|\mathrm{F}\left(s, \vartheta(s), \int_{0}^{s} \varrho(s, \tau, \vartheta(\tau)) d \tau\right)+\mathrm{F}(s, 0,0)-\mathrm{F}(s, 0,0)\right\| d s
\end{aligned}
$$


$\leq \quad\left\|\mathrm{R}\left(t_{2}\right) \vartheta_{0}-\mathrm{R}\left(t_{1}\right) \vartheta_{0}\right\|$

$$
\begin{aligned}
& +\int_{0}^{t_{1}}\left\|\mathrm{R}\left(t_{2}-s\right)-\mathrm{R}\left(t_{1}-s\right)\right\|_{\mathscr{L}(\mathbf{W})} \\
& \times\left(f(s)\left[\|\vartheta(s)\|+\left\|\int_{0}^{s} \varrho(s, \tau, \vartheta(\tau)) d \tau\right\|+1\right]+\|\mathrm{F}(s, 0,0)\|\right) d s \\
& +\int_{t_{1}}^{t_{2}}\left\|\mathrm{R}\left(t_{2}-s\right)\right\|\left(f(s)\left[\|\vartheta(s)\|+\left\|\int_{0}^{s} \varrho(s, \tau, \vartheta(\tau)) d \tau\right\|+1\right]+\|\mathrm{F}(s, 0,0)\|\right) d s \\
& \leq \quad\left\|\mathrm{R}\left(t_{2}\right) \vartheta_{0}-\mathrm{R}\left(t_{1}\right) \vartheta_{0}\right\| \\
& +\int_{0}^{t_{1}}\left\|\mathrm{R}\left(t_{2}-s\right)-\mathrm{R}\left(t_{1}-s\right)\right\|_{\mathscr{L}(\mathbf{W})} \\
& \times\left(f(s)\left[q+\left\|\int_{0}^{s} \varrho(s, \tau, \vartheta(\tau)) d \tau\right\|+1\right]+\|\mathrm{F}(s, 0,0)\|\right) d s \\
& +\int_{t_{1}}^{t_{2}}\left\|\mathrm{R}\left(t_{2}-s\right)\right\|\left(f(s)\left[q+\left\|\int_{0}^{s} \varrho(s, \tau, \vartheta(\tau)) d \tau\right\|+1\right]+\|\mathrm{F}(s, 0,0)\|\right) d s \\
& \leq\left\|\mathrm{R}\left(t_{2}\right) \vartheta_{0}-\mathrm{R}\left(t_{1}\right) \vartheta_{0}\right\|+\int_{0}^{t_{1}}\left\|\mathrm{R}\left(t_{2}-s\right)-\mathrm{R}\left(t_{1}-s\right)\right\|_{\mathscr{L}(\mathbf{w})}\|\mathrm{F}(s, 0,0)\| d s \\
& +\int_{0}^{t_{1}}\left\|\mathrm{R}\left(t_{2}-s\right)-\mathrm{R}\left(t_{1}-s\right)\right\|_{\mathscr{L}(\mathbf{W})} f(s)\left(1+q+q \int_{0}^{s} g(\tau) d \tau\right) d s \\
& +\int_{0}^{t_{1}} \int_{0}^{s}\left\|\mathrm{R}\left(t_{2}-s\right)-\mathrm{R}\left(t_{1}-s\right)\right\|_{\mathscr{L}(\mathbf{W})} f(s)\|\varrho(s, \tau, 0)\| d \tau d s \\
& +\mathrm{m}_{0} \int_{t_{1}}^{t_{2}} e^{-\gamma\left(t_{2}-s\right)} f(s) d s+\mathrm{m}_{0} q \int_{t_{1}}^{t_{2}} e^{-\gamma\left(t_{2}-s\right)} f(s)\left(1+\int_{0}^{s} g(\tau) d \tau\right) d s \\
& +\mathrm{m}_{0} \int_{t_{1}}^{t_{2}} \int_{0}^{s} e^{-\gamma\left(t_{2}-s\right)} f(s)\|\varrho(s, \tau, 0)\| d \tau d s+\mathrm{m}_{0} \int_{t_{1}}^{t_{2}} e^{-\gamma\left(t_{2}-s\right)}\|\mathrm{F}(s, 0,0)\| d s .
\end{aligned}
$$

By the continuity of $(R(t))_{t \geq 0}$ in the operator-norm topology and the dominated convergence theorem, we conclude that the right hand side of the above inequality tends to zero and independent of $\vartheta$ as $t_{2} \rightarrow t_{1}$. Hence $\Gamma\left(B_{q}\right)$ is equicontinuous.

Consider the measure of noncompactness $\Xi(\mathrm{K})$ defined on the family of bounded subset of $\mathrm{BC}(\mathrm{I}, \mathbf{W})$ by

$$
\Xi(\mathrm{K})=\omega_{0}^{T}(\mathrm{~K})+\sup _{t \in I} e^{-n_{0} \sigma(t)} \bar{\alpha}(\mathrm{K}(t))+\lim _{t \rightarrow+\infty} \sup _{t \in I}\|\vartheta(t)\|,
$$


where

$$
\sigma(t)=2 \mathrm{~m}_{0} \int_{0}^{t}\left(\omega_{1}(s)+2 \omega_{2}(s) M^{\star} s\right) d s, \quad n_{0}>1, \quad \bar{\alpha}(\mathrm{K}(t))=\sup _{0 \leq r \leq t} \alpha(\mathrm{K}(r)) .
$$

Step 4 We show that the Mönch conditions hold.

Suppose $\mathrm{K} \subset \mathrm{BC}(\mathrm{I}, \mathbf{W})$ is countable and $K \subset \overline{\operatorname{Conv}}(\{0\} \cup \Gamma(\mathrm{K}))$.

Claim 1. $\omega_{0}^{T}(\mathrm{~K})=0$.

Using the properties of $\omega_{0}^{T}($.$) (see[29]), and the fact that \Gamma\left(B_{q}\right)$ is equicontinuous, we get

$$
\omega_{0}^{T}(\mathrm{~K}) \leq \omega_{0}^{T}(\overline{\operatorname{Conv}}(\{0\} \cup \Gamma(\mathrm{K}(t))))=\omega_{0}^{T}(\Gamma(\mathrm{K}(t)))=0 .
$$

Hence we deduce that

$$
\omega_{0}^{T}(\mathrm{~K})=0 .
$$

Let assume that $\mathrm{K}=\left\{\vartheta^{(n)}\right\}_{n \geq 1}$. Since $\Gamma$ maps $B_{q}$ into an equicontinuous family, $\Gamma(\mathrm{K})$ is also equicontinuous on I.

Claim 2. $\sup _{t \in I} e^{-n_{0} \sigma(t)} \bar{\alpha}(\mathrm{K}(t))=0$.

Using the properties of $\alpha$, Lemma 2.5 and hypotheses $\left(\mathbf{C}_{1}\right),\left(\mathbf{C}_{2}\right),\left(\mathbf{C}_{3}\right)$ and $\left(\mathbf{C}_{4}\right)$, we get

$$
\begin{aligned}
\alpha(\mathrm{K}(t)) & \leq \alpha(\overline{\operatorname{Conv}}(\{0\} \cup \Gamma(\mathrm{K}(t))))=\alpha(\Gamma(\mathrm{K}(t))) \\
& \leq \alpha\left(\int_{0}^{t} \mathrm{R}(t-s) \mathrm{F}\left(s, \mathrm{~K}(s), \int_{0}^{s} \varrho(s, \tau, \mathrm{K}(\tau)) d \tau\right) d s\right) \\
& \leq 2 \mathrm{~m}_{0} \int_{0}^{t} \alpha\left(\mathrm{F}\left(s, \mathrm{~K}(s), \int_{0}^{s} \varrho(s, \tau, \mathrm{K}(\tau)) d \tau\right)\right) d s \\
& \leq 2 \mathrm{~m}_{0} \int_{0}^{t}\left[\omega_{1}(s) \alpha(\mathrm{K}(s))+\omega_{2}(s) \alpha\left(\int_{0}^{s} \varrho(s, \tau, \mathrm{K}(\tau)) d \tau\right)\right] d s \\
& \leq 2 \mathrm{~m}_{0} \int_{0}^{t}\left[\mathrm{w}_{1}(s) \alpha(\mathrm{K}(s))+2 \omega_{2}(s) M^{\star} \int_{0}^{s} \alpha(\mathrm{K}(\tau)) d \tau\right] d s \\
& \leq 2 \mathrm{~m}_{0} \int_{0}^{t} \omega_{1}(s) \sup _{0 \leq s \leq t} \alpha(\mathrm{K}(s)) d s+4 \mathrm{~m}_{0} \int_{0}^{t} \omega_{2}(s) M^{\star} s \sup _{0 \leq \tau \leq s} \alpha(\mathrm{K}(\tau)) d s .
\end{aligned}
$$

Therefore, we have

$$
\alpha(\mathrm{K}(t)) \leq 2 \mathrm{~m}_{0} \int_{0}^{t}\left(\omega_{1}(s)+2 \omega_{2}(s) M^{\star} s\right) e^{n_{0} \sigma(s)} \times \sup _{t \in I} e^{-n_{0} \sigma(s)} \sup _{0 \leq s \leq t} \bar{\alpha}(\mathrm{K}(s)) d s,
$$

then

$$
e^{-n_{0} \sigma(t)} \alpha(\mathrm{K}(t)) \leq \frac{1}{n_{0}} \sup _{t \in I} e^{-n_{0} \sigma(t)} \bar{\alpha}(\mathrm{K}(t))
$$

hence

$$
e^{-n_{0} \sigma(t)} \sup _{t \in I} \alpha(\mathrm{K}(t)) \leq \frac{1}{n_{0}} \sup _{t \in I} e^{-n_{0} \sigma(t)} \bar{\alpha}(\mathrm{K}(t)) .
$$

Since $e^{-n_{0} \sigma(t)} \sup _{0 \leq s \leq t} \alpha(\mathrm{K}(t)) \leq e^{-n_{0} \sigma(t)} \sup _{t \in I} \alpha(\mathrm{K}(t))$, 
we get

$$
\begin{aligned}
e^{-n_{0} \sigma(t)} \sup _{0 \leq r \leq t} \alpha(\mathrm{K}(r)) & \leq \frac{1}{n_{0}} \sup _{t \in I} e^{-n_{0} \sigma(t)} \bar{\alpha}(\mathrm{K}(t)), \\
\sup _{t \in I} e^{-n_{0} \sigma(t)} \sup _{0 \leq r \leq t} \alpha(\mathrm{K}(r)) & \leq \frac{1}{n_{0}} \sup _{t \in I} e^{-n_{0} \sigma(t)} \bar{\alpha}(\mathrm{K}(t)) .
\end{aligned}
$$

Then

$$
\sup _{t \in I} e^{-n_{0} \sigma(t)} \bar{\alpha}(\mathrm{K}(t)) \leq \frac{1}{n_{0}} \sup _{t \in I} e^{-n_{0} \sigma(t)} \bar{\alpha}(\mathrm{K}(r)) .
$$

Since $n_{0}>1$, then

$$
\sup _{t \in I} e^{-n_{0} \sigma(t)} \bar{\alpha}(\mathrm{K}(r))=0 .
$$

Claim 3. $\lim _{t \rightarrow+\infty} \sup _{t \in I}\|\vartheta(t)\|=0$.

We have

$$
\begin{aligned}
& \|\vartheta(t)\| \leq \mathrm{m}_{0} e^{-\gamma t}\left\|\vartheta_{0}\right\|+\mathrm{m}_{0} \int_{0}^{t} e^{-\gamma(t-s)} \mathrm{f}(s)(\|\vartheta(s)\|) d s \\
& +\mathrm{m}_{0} \int_{0}^{t} e^{-\gamma(t-s)} \mathrm{f}(s) \int_{0}^{s} g(\tau)(\|\vartheta(\tau)\|) d \tau d s \\
& +\mathrm{m}_{0} \int_{0}^{t} e^{-\gamma(t-s)} \mathrm{f}(s) \int_{0}^{s} M e^{-\omega(s-\tau)} d \tau d s \\
& +\mathrm{m}_{0} \int_{0}^{t} e^{-\gamma(t-s)} \mathrm{f}(s) d s+\mathrm{m}_{0} \int_{0}^{t} e^{-\gamma(t-s)}\|\mathrm{F}(s, 0,0)\| d s \\
& \leq \mathrm{m}_{0} e^{-\gamma t}\left\|\vartheta_{0}\right\|+\mathrm{m}_{0} \int_{0}^{t} e^{-\gamma(t-s)} \mathrm{f}(s)(\|\vartheta(s)\|) d s \\
& +\mathrm{m}_{0} \int_{0}^{t} e^{-\gamma(t-s)} \mathrm{f}(s) \int_{0}^{s} g(\tau)(\|\vartheta(\tau)\|) d \tau d s+\mathrm{m}_{0}\left(1+\frac{M}{\omega}\right) \int_{0}^{t} e^{-\gamma(t-s)} \mathrm{f}(s) d s \\
& +\mathrm{m}_{0} \int_{0}^{t} e^{-\gamma(t-s)}\|\mathrm{F}(s, 0,0)\| d s \\
& \leq \mathrm{m}_{0} e^{-\gamma t}\left\|\vartheta_{0}\right\|+\mathrm{m}_{0} \int_{0}^{t} e^{-\gamma(t-s)} \mathrm{f}(s)\left[\|\vartheta(s)\|+\int_{0}^{s} g(\tau)\|\vartheta(\tau)\| d \tau\right] d s \\
& +\mathrm{m}_{0}\left(1+\frac{M}{\omega}\right) \int_{0}^{t} e^{-\gamma(t-s)} \mathrm{f}(s) d s+\mathrm{m}_{0} \int_{0}^{t} e^{-\gamma(t-s)}\|\mathrm{F}(s, 0,0)\| d s .
\end{aligned}
$$

By Lemma 2.6, we have

$$
\begin{aligned}
& \|\vartheta(t)\| \\
& \leq \quad B(t)+\mathrm{m}_{0} \int_{0}^{t} e^{-\gamma(t-s)} \mathrm{f}(s) \\
& \quad \times\left[B(s)+\int_{0}^{s} B(\tau)\left(\mathrm{m}_{0} e^{-\gamma(t-\tau)} \mathrm{f}(\tau)+g(\tau)\right) \times \exp \left(\int_{\tau}^{s}\left(\mathrm{~m}_{0} e^{-\gamma(t-\delta)} \mathrm{f}(\delta)+g(\delta)\right) d \delta\right) d \tau\right] d s,
\end{aligned}
$$

where

$$
B(t)=\mathrm{m}_{0} e^{-\gamma t}\left\|\vartheta_{0}\right\|+\mathrm{m}_{0}\left(1+\frac{M}{\omega}\right) \int_{0}^{t} e^{-\gamma(t-s)} \mathrm{f}(s) d s+\mathrm{m}_{0} \int_{0}^{t} e^{-\gamma(t-s)}\|\mathrm{F}(s, 0,0)\| d s .
$$


Then,

$$
\|\vartheta(t)\| \leq B(t)+\Lambda_{0} \int_{0}^{t} e^{-\gamma(t-s)} \mathbf{f}(s) d s,
$$

where $\Lambda_{0}=\left[\mathrm{m}_{0}\left\|\vartheta_{0}\right\|+\mathrm{m}_{0} \overline{\mathrm{F}}+\mathrm{m}_{0} \overline{\mathrm{f}}\left(1+\frac{M}{\omega}\right)\right]\left[1+\overline{\mathrm{f}}\left(\mathrm{m}_{0} \overline{\mathrm{f}}+\|g\|_{L^{1}}\right)\right] \exp \left(\mathrm{m}_{0} \overline{\mathrm{f}}+\|g\|_{L^{1}}\right)$. Hence, by conditions $\left(\mathbf{C}_{3}\right)$ and $\left(\mathbf{C}_{4}\right)$, it follows that

$$
\lim _{t \rightarrow+\infty} \sup _{t \in I}\|\vartheta(t)\|=0 .
$$

From Claims, 1, 2,3, we obtain

$$
\Xi(K)=0
$$

Thus we find that $\mathrm{K}$ is relatively compact.

Step 5. A priori bounds. We now show there exists an open set $\mathscr{O} \subset \mathrm{BC}(\mathrm{I}, \mathbf{W})$ with $\vartheta \neq \kappa \Gamma \vartheta$, for $\kappa \in(0,1)$ and $\vartheta \in \partial \mathscr{O}$. Let $\vartheta \in \mathrm{BC}(\mathrm{I}, \mathrm{W})$ and $\vartheta=\kappa \Gamma \vartheta$ for some $\kappa \in(0,1)$. Then by (3.9), we get

$$
\begin{aligned}
\|\vartheta(t)\| & \leq B(t)+\Lambda_{0} \mathrm{~m}_{0} \int_{0}^{t} e^{-\gamma(t-s)} \mathrm{f}(s) d s \\
& \leq \mathrm{m}_{0}\left\|\vartheta_{0}\right\|+\mathrm{m}_{0}\left(1+\frac{M}{\omega}\right) \overline{\mathrm{f}}+\mathrm{m}_{0} \overline{\mathrm{F}}+\Lambda_{0} \mathrm{~m}_{0} \overline{\mathrm{f}}=\mathrm{H} .
\end{aligned}
$$

Set

$$
\mathscr{O}=\{\vartheta \in \mathrm{K}:\|\vartheta\|<\mathrm{H}+1\} .
$$

By the choice of $\mathscr{O}$, there is no $\vartheta \in \partial \mathscr{O}$ such that $\vartheta=\kappa \Gamma \vartheta$, for $\kappa \in(0,1)$. Thus by Mönch fixed point theorem, the operator $\Gamma: \overline{\mathscr{O}} \rightarrow \mathrm{BC}(\mathrm{I}, \mathrm{W})$ has at least one fixed point $\vartheta$ in $\overline{\mathscr{O}}$ which is a mild solution of system (1.1).

\section{Uniform local attractivity of mild solutions}

The aim of this section is to investigate the uniform local attractivity of solutions of problem (1.1).

Denote by $\bar{B}\left(\vartheta^{\star}, \Delta_{0}\right)$ the closed ball in $\mathrm{BC}(\mathrm{I}, \mathbf{W})$, where $\vartheta^{\star}$ is a solution of (1.1) and $\Delta_{0}$ satisfies

$$
\Delta_{0} \geq \frac{\mathrm{m}_{0} \overline{\mathrm{f}}}{1-\mathrm{m}_{0} \overline{\mathrm{f}}\left(1+\|g\|_{L^{1}}\right)} .
$$

Then for $\vartheta \in \bar{B}\left(\vartheta^{\star}, \Delta_{0}\right)$, by $\left(\mathbf{C}_{1}\right)-\left(\mathbf{C}_{3}\right)$ and (4.1) we have

$$
\begin{aligned}
& \left\|\Gamma \vartheta(t)-\vartheta^{\star}(t)\right\|=\left\|\Gamma \vartheta(t)-\Gamma \vartheta^{\star}(t)\right\| \\
\leq & \| \int_{0}^{t} \mathrm{R}(t-s) \mathrm{F}\left(s, \vartheta(s), \int_{0}^{s} \varrho(s, \tau, \vartheta(\tau)) d \tau\right) d s \\
& -\int_{0}^{t} \mathrm{R}(t-s) \mathrm{F}\left(s, \vartheta^{\star}(s), \int_{0}^{s} \varrho\left(s, \tau, \vartheta^{\star}(\tau)\right) d \tau\right) d s \| \\
\leq & \left\|\int_{0}^{t} \mathrm{R}(t-s)\left[\mathrm{F}\left(s, \vartheta(s), \int_{0}^{s} \varrho(s, \tau, \vartheta(\tau)) d \tau\right)-\mathrm{F}\left(s, \vartheta^{\star}(s), \int_{0}^{s} \varrho\left(s, \tau, \vartheta^{\star}(\tau)\right) d \tau\right)\right] d s\right\|
\end{aligned}
$$




$$
\begin{aligned}
& \leq \mathrm{m}_{0} \int_{0}^{t} e^{-\gamma(t-s)} f(s)\left[1+\left\|\vartheta(s)-\vartheta^{\star}(s)\right\|+\int_{0}^{s}\left\|\varrho(s, \tau, \vartheta(\tau))-\varrho\left(s, \tau, \vartheta^{\star}(\tau)\right)\right\| d \tau\right] d s \\
& \leq \mathrm{m}_{0} \int_{0}^{t} e^{-\gamma(t-s)} f(s)\left[1+\left\|\vartheta(s)-\vartheta^{\star}(s)\right\|+\int_{0}^{s} g(\tau)\left\|\vartheta(\tau)-\vartheta^{\star}(\tau)\right\| d \tau\right] d s \\
& \leq \mathrm{m}_{0} \int_{0}^{t} e^{-\gamma(t-s)} f(s)\left[1+\left\|\vartheta(s)-\vartheta^{\star}(s)\right\|+\int_{0}^{s} g(\tau)\left(\left\|\vartheta(\tau)-\vartheta^{\star}(\tau)\right\|\right) d \tau\right] d s \\
& \leq \mathrm{m}_{0} \int_{0}^{t} e^{-\gamma(t-s)} f(s) d s+\mathrm{m}_{0} \int_{0}^{t} e^{-\gamma(t-s)} f(s)\left\|\vartheta(s)-\vartheta^{\star}(s)\right\| d s \\
& +\mathrm{m}_{0} \int_{0}^{t} e^{-\gamma(t-s)} f(s) \int_{0}^{s} g(\tau)\left\|\vartheta(\tau)-\vartheta^{\star}(\tau)\right\| d \tau d s \\
& \leq \mathrm{m}_{0} \overline{\mathrm{f}}+\mathrm{m}_{0} \overline{\mathrm{f}} \Delta_{0}+\mathrm{m}_{0} \overline{\mathrm{f}}\|g\|_{L^{1}} \Delta_{0} \\
& \leq \mathrm{m}_{0} \overline{\mathrm{f}}+\mathrm{m}_{0} \overline{\mathrm{f}}\left(1+\|g\|_{L^{1}}\right) \Delta_{0} \\
& \leq \Delta_{0} \text {. }
\end{aligned}
$$

Therefore, we get $\Gamma\left(\bar{B}\left(\vartheta^{\star}, \Delta_{0}\right)\right) \subset \bar{B}\left(\vartheta^{\star}, \Delta_{0}\right)$.

So, for any solutions $\vartheta^{(1)}, \vartheta^{(2)} \in \bar{B}\left(\vartheta^{\star}, \Delta_{0}\right)$ to problem (1.1) and $t \in I$, we have

$$
\begin{aligned}
& \left\|\Gamma \vartheta^{(1)}(t)-\Gamma \vartheta^{(2)}(t)\right\| \\
\leq & \mathrm{m}_{0} \int_{0}^{t} e^{-\gamma(t-s)} f(s)\left[1+\left\|\vartheta^{(1)}(s)-\vartheta^{(2)}(s)\right\|+\int_{0}^{s} g(\tau)\left\|\vartheta^{(1)}(\tau)-\vartheta^{(2)}(\tau)\right\| d \tau\right] d s \\
\leq & \mathrm{m}_{0} \int_{0}^{t} e^{-\gamma(t-s)} f(s)\left[1+\left\|\vartheta^{(1)}(s)-\vartheta^{(2)}(s)\right\|+\int_{0}^{s} g(\tau)\left(\left\|\vartheta^{(1)}(\tau)-\vartheta^{(2)}(\tau)\right\|\right) d \tau\right] d s \\
\leq & \mathrm{m}_{0} \int_{0}^{t} e^{-\gamma(t-s)} f(s)\left[1+\Delta_{0}+\|g\|_{L^{1}} \Delta_{0}\right] d s \\
\leq & \mathrm{m}_{0}\left[1+\Delta_{0}\left(1+\|g\|_{L^{1}}\right)\right] \int_{0}^{t} e^{-\gamma(t-s)} f(s) d s .
\end{aligned}
$$

Hence, from $\left(\mathbf{C}_{3}\right)$ - (ii), we conclude that for all $\epsilon>0$, there are real numbers $T>0$ such that

$$
\mathrm{m}_{0} \int_{0}^{t} e^{-\gamma(t-s)} f(s) d s \leq \frac{\epsilon}{\mathrm{m}_{0}\left[1+\Delta_{0}\left(1+\|g\|_{L^{1}}\right)\right]} \text { for all } t \geq T .
$$

Then from the above inequality, it follows that

$$
\left\|\vartheta^{(1)}(t)-\vartheta^{(2)}(t)\right\| \leq \epsilon \text { for all } t \geq T .
$$

This implies the uniform local attractivity of solutions of the integro-differential equation (1.1). 


\section{Example}

We consider the following class of partial integro-differential system:

$$
\left\{\begin{aligned}
\frac{\partial}{\partial t} v(t, \xi)= & {\left[\frac{\partial^{2} v(t, \xi)}{\partial \xi^{2}}+\tilde{b} \frac{\partial v(t, \xi)}{\partial \xi}+\tilde{c} v(t, \xi)\right]+\int_{0}^{t} \Xi(t-s)\left[\frac{\partial^{2} x(s, \xi)}{\partial \xi^{2}}+\tilde{b} \frac{\partial x(s, \xi)}{\partial \xi}+\tilde{c} x(s, \xi)\right] d s } \\
+ & \frac{\sin (t) e^{-|v(t, \xi)|-\gamma t}}{\left(t^{2}+1\right)}+\frac{\ln \left(1+e^{-\gamma t}\right) v(t, \xi)}{\left(t^{2}+1\right)(1+|v(t, \xi)|)} \\
& +\frac{\sin \left(e^{-\gamma t}\right)}{\left(t^{2}+1\right)} \int_{0}^{t} \frac{\ln (1+t) \cos (v(s, \xi)) e^{-\gamma(t-s)}}{1+2 t^{2}+s^{2}} d s, \quad t \in I, \quad \xi \in[0,1], \\
v(t, 0) \quad & v(t, 1)=0, \quad \text { for } t \geq 0,
\end{aligned}\right.
$$

where $\Xi: \mathbb{R}^{+} \mapsto \mathbb{R}$ is continuous, $\tilde{b}, \tilde{c} \in \mathbb{R}$.

Let $\mathbf{W}=L^{2}(0,1)$. We define the operator $A$ induced on $\mathbf{W}$ as follows:

$$
\left\{\begin{array}{l}
D(\mathrm{~A})=H^{2}(0,1) \cap H_{0}^{1}(0,1) \\
\mathrm{A} z=z^{\prime \prime}+\tilde{b} z+\tilde{c} z, \tilde{b}, \tilde{c} \in \mathbb{R} .
\end{array}\right.
$$

From [24, p. 173], we know that A is the infinitesimal generator of an analytic $C_{0}$ semigroup $(T(t))_{t \geq 0}$ on $\mathbf{W}$. Since the semigroup generated by $A$ is analytic, then it is norm continuous for $t>0$.Thus by Theorem 2.3 the corresponding resolvent operator is operator-norm continuous for $t>0$.

We define the operators $Y(t): Y \mapsto X$ as follows: $Y(t) N=\Xi(t)$ AN for $t \geq 0$ and $N \in D(\mathrm{~A})$.

We put $\vartheta(t)(\xi)=v(t, \xi)$ for $t \in[0,+\infty)$, and define

$$
\begin{aligned}
\mathrm{F}(t, \Phi, \Psi)(\xi) & =\frac{\sin (t) e^{-|\Phi(t, \xi)|-\gamma t}}{\left(t^{2}+1\right)}+\frac{\ln \left(1+e^{-\gamma t}\right)}{\left(t^{2}+1\right)} \frac{\Phi(t, \xi)}{(1+|\Phi(t, \xi)|)}+\frac{\sin \left(e^{\gamma t}\right)}{\left(t^{2}+1\right)} \Psi(t, \xi) \\
\rho(t, s, \Phi)(\xi) & =\frac{\ln \left(2 t+e^{-s}\right) \cos (\Phi(s, \xi)) e^{-\gamma(t-s)}}{\left(1+2 t^{2}+s^{2}\right)^{3}}
\end{aligned}
$$

Then, equation (5.1) takes the following abstract form

$$
\left\{\begin{array}{l}
\vartheta^{\prime}(t)=\mathrm{A} \vartheta(t)+\int_{0}^{t} Y(t-r) \vartheta(r) d r+\mathrm{F}\left(t, \vartheta(t), \int_{0}^{t} \rho(t, r, \vartheta(r)) d r\right), t \in I=[0,+\infty) \\
\vartheta(0)=\vartheta_{0} .
\end{array}\right.
$$

We suppose $\Xi$ is a bounded and $C^{1}$ function such that $\Xi$ is bounded and uniformly continuous. Accordingly, the assumptions $\left(\mathbf{H}_{\mathbf{1}}\right)$ and $\left(\mathbf{H}_{\mathbf{1}}\right)$ are fulfilled. Thus from Theorem 2.1 and Theorem 2.3, the problem (5.1) has a resolvent operator $(\mathrm{R}(t))_{t \geq 0}$ on $\mathrm{W}$ which is norm continuous for $t>0$.

We assume moreover that there exists $\beta_{1}>a>1$ and $\Xi(t)<\frac{1}{a} e^{-\beta_{1} t}$ for all $t \geq 0$. Thanks to Lemma 5.2 in [32], we have the following estimates $\|\mathrm{R}(t)\| \leq e^{-\lambda t}$ where $\lambda=\left(1-\frac{1}{a}\right)$. Hence (C2) hold with $\mathrm{m}_{0}=1$ and $\gamma=1-\frac{1}{a}$.

Moreover, applying the inequalities

$$
\ln (1+x) \leq x, \quad \sin x \leq x,
$$

we have

$$
\left\|\mathrm{F}\left(t, \Phi_{2}, \Psi_{2}\right)(\xi)-\mathrm{F}\left(t, \Phi_{1}, \Psi_{1}\right)(\xi)\right\| \leq \frac{e^{-\gamma t}}{t^{2}+1}\left(1+\left\|\Phi_{2}(t, \xi)-\Phi_{1}(t, \xi)\right\|+\left\|\Psi_{2}(t, \xi)-\Psi_{1}(t, \xi)\right\|\right)
$$


and

$$
\left\|\varrho\left(t, s, \Phi_{2}\right)-\varrho\left(t, s, \Phi_{1}\right)\right\| \leq \frac{\ln (1+2 t)}{\left(t^{2}+1\right)^{3}}\left\|\Phi_{2}-\Phi_{1}\right\| .
$$

Hence conditions $\left(\mathbf{C}_{3}\right)-i$ and $\left(\mathbf{C}_{4}\right)-i$ are satisfied with $f(t)=\frac{e^{-\gamma t}}{t^{2}+1}, g(t)=\frac{\ln (1+2 t)}{\left(t^{2}+1\right)^{3}}$.

Also, we have

$$
\begin{aligned}
\int_{0}^{t} e^{-\gamma(t-r)} \mathrm{F}(r, 0,0) d r & =\int_{0}^{t} e^{-\gamma(t-r)} \frac{\sin (r) e^{-\gamma r}}{r^{2}+1} d r \\
& \leq e^{-\gamma t} \arctan (t) \rightarrow 0 \text { as } t \rightarrow+\infty
\end{aligned}
$$

and

$$
\begin{aligned}
\|\varrho(t, s, 0)\| & \leq \frac{2 t}{\left(1+t^{2}\right)^{3}} e^{-\gamma(t-s)} \\
& \leq \frac{25 \sqrt{5}}{108} e^{-\gamma(t-s)} .
\end{aligned}
$$

By (5.3), for any bounded subsets $E_{1}, E_{2} \subset \mathrm{W}$, we get

$$
\alpha\left(\mathrm{F}\left(t, E_{1}, E_{2}\right)\right) \leq \frac{\ln \left(1+e^{-\gamma t}\right)}{\left(t^{2}+1\right)} \alpha\left(E_{1}\right)+\frac{\sin \left(e^{-\gamma t}\right)}{\left(t^{2}+1\right)} \alpha\left(E_{1}\right) \text { for a.e } t \in I .
$$

By (5.6), for any bounded subsets $E \subset$ W, we get

$$
\alpha(\varrho(t, s, E))=\frac{25 \sqrt{5}}{108} \alpha(E) .
$$

Hence $\left(\mathbf{C}_{3}\right)-i i i$ and $\left(\mathbf{C}_{4}\right)-i i i$ are satisfied

Consequently, all the conditions of theorem (3.1) are fullfilled and the problem (5.1) admits mild solutions which are uniformly locally attractive.

\section{References}

[1] A. Belleni-Morante: An integrodifferential equation arising from the theory of heat conduction in rigid material with memory, Boll. Un. Mat. Ital. 15 (1978), 470-482.

[2] A. Belleni-Morante and G. F. Roach: A mathematical model for Gamma ray transport in the cardiac region, J. Math. Anal. Appl. 244 (2000), 498-514.

[3] A. Baliki, M. Benchohra and J. Graef: Global existence and stability for second order functional evolution equations with infinite delay. Electron. J. Qual. Theory Differ. Equ. (2016), 1-10.

[4] A. Jawahdou: Mild solutions of functional semilinear evolution Volterra integrodifferential equations on an unbounded interval, Nonlinear Anal. 74 (2011), 7325-7332.

[5] B.G. Pachpatte: Inequalities for diferential and integral equations. Academic Press, Inc., SanDiego, CA, (1998).

[6] D. Tang and M. Samuel Rankin III: Peristaltic transport of a heat conducting viscous fluid as an application of abstract differential equations and semigroup of operators, J. Math. Anal. Appl. 169 (1992), 391-407.

[7] G. Marino, P. Pietramala and H.K. Xu: Nonlinear neutral integrodifferential equations on unbounded intervals. Int. Math.1, No. 17-20, (2006), 933-946.

[8] H. Mönch: Boundary value problems for nonlinear ordinary differential equations of second order in Banach spaces. Nonlinear Anal. Theory Methods Appl.4(5), (1980), 985-999.

[9] J. Wu:Theory and Application of Partial Functional Differential Equations, Springer-Verlag, New York, (1996).

[10] M.A. Darwish, On quadratic integral equation of fractional orders, J. Math, Anal. Appl. 311 (2005) 112-119.

[11] M. Mursaleen, A. Alotaibi, Infinite system of differential equations in some spaces. Abstr. Appl. Anal. (2012) doi:10.1155/2012/863483

[12] J. Banas and K. Goebel: Measure of Noncompactness in Banach Spaces. Lecture Notes in Pure and Applied Mathematics. Dekker, New York (1980). 
[13] J.Banaś and B.C.Dhage:Global asymptotic stability of solutions of a functional integral equation, Nonlinear Anal., 69 (2008), 1945-1952.

[14] J. Banaś and B. Rzepka,:On existence and asymptotic stability of solutions of a nonlinear integral equation, J. Math. Anal. Appl., 284 (2003), 165-173.

[15] R.P. Agarwal, D. OíRegan, Singular Volterra integral equations. Appl. Math. Lett. 13(2000)115-120.

[16] M. Benchohra, D. Seba, Integral equations of fractional order with multiple time delays in Banach spaces, Electron. J. Differential Equations 56(2012)1-8.

[17] J. Banaś and T. Zajac: Solvability of a functional integral equation of fractional order in the class of functions having limits at infinity, Nonlinear Anal., 71 (2009), 5491-5500.

[18] J. Blot, C. Buse and P. Cieutat: Local attractivity in nonautonomous semilinear evolution equations. Nonauton. Dyn.Syst. 1 (2014), 72-82.

[19] J. Liang, J.H Liu and T.J Xiao: Nonlocal problems for integrodifferential equations. Dynamics of Continuous, Discrete and Impulsive Systems, 15, 815-824 (2008).

[20] J. Liang and T.J Xiao: Semilinear integrodifferential equations with nonlocal initial conditions. Computers \& Mathematics with Applications, 47, (6-7), 863-875 (2004).

[21] J. Blot, C. Buse and P. Cieutat: Local attractivity in nonautonomous semilinear evolution equations. Nonauton. Dyn. Syst. 1 , (2014), 72-82.

[22] J.R Wang, Z. Fan and Y. Zhou: Nonlocal controllability of semilinear dynamic systems with fractional derivative in Banach spaces. J. Optim. Theory Appl. 154 , (2012), 292-302.

[23] K. Balachandran and R. Sakthivel :Controllability of integrodifferential systems in Banach spaces . Applied Mathematics and Computation , 118 (2001), 63-71.

[24] K.J Engel and R. Nagel:One-parameter semigroups for linear evolution equationsSpringer-Verlag, New York (2000) .

[25] K. Ezzinbi, G. Degla and P. Ndambomve: Controllability for some partial functional integrodifferential equations with nonlocal condition in Banach spaces. Discussiones Mathematicae Differ. Incl. Control Optim. 35 (1) (2015), 1-22 .

[26] K. Ezzinbi and S. Ghnimi:Existence and regularity of solutions for neutral partial functional integrodifferential equations. Nonlinear Analysis: Real World Applications ; 11 (2010), 2335-2344.

[27] K.H Bete, C. Ogouyandjoua, Am. Diop and M.A. Diop: On the attractivity of an integrodifferential system with state-dependent delay, J. Nonlinear Sci.Appl., 12 (2019), 611-620.

[28] K. Kuratowski, Sur les espaces complets, Fund. Math. 5(1930)301-309.

[29] L.Olszowy: On existence of solutions of a quadratic Urysohn integral equation on an unbounded interval, Comment.Math. 46 (2008), 103-112.

[30] M.A Diop, K. Ezzinbi and M. Lo:Existence and uniqueness of mild solutions to some neutral stochastic partial functional integrodifferential equations with non-Lipschitz coefficients, Vol.2012, Hindawi Publishing Corporation, (2012), 1-12.

[31] M. Benchohra and N. Rezoug: Existence and attractivity of solutions of semilinear Volterra type integrodifferential evolution equations, Surveys in Mathematics and its Applications 13 (2018), 215-235

[32] M. Dieye, M. A Diop and K. Ezzinbi, Controllability for some integrodifferential equations driven by vector measures, Math.Meth.Appl.Sci.2016, (wileyonlinelibrary.com) DOI:10.1002/mma.4125.(2016).

[33] N. U. Ahmed:Semigroup Theory with Applications to Systems and Control, Harlow John Wiley and Sons, Inc., New York, (1991).

[34] P. Aviles and J. Sandefur: Nonlinear second order equations with applications to partial differential equations, J. Differential Equations 58 (1985), 404-427.

[35] R. P. Agarwal and D. O'Regan: Infinite interval problems modelling the flow of a gas through a semi-infinite porous medium. Stud. Appl. Math. 108 (2002), 245-257.

[36] R. P. Agarwal and D. O'Regan: Infinite interval problems modeling phenomena which arise in the theory of plasma and electrical potential theory, Stud. Appl. Math. 111 (2003), no. 3, 339-358.

[37] R. P. Agarwal, A. Domoshnitsky, rm and Ya. Goltser: Stability of partial functional integro-differential equations. J. Dyn. Control Syst. 12 (2006), No. 1, 1-31.

[38] R. C. Grimmer, Resolvent operators for integral equations in a Banach space, Transactions of the American Mathematical Society, 273 (1982), 333-349.

[39] S. Abbas and M. Benchohra:Advanced Functional Evolution Equations and Inclusions, Springer, Cham, (2015). 